\title{
UNCERTAINTY QUANTIFICATION AND WEAK APPROXIMATION OF AN ELLIPTIC INVERSE PROBLEM*
}

\author{
M. DASHTI ${ }^{\dagger}$ AND A. M. STUART ${ }^{\dagger}$
}

\begin{abstract}
We consider the inverse problem of determining the permeability from the pressure in a Darcy model of flow in a porous medium. Mathematically the problem is to find the diffusion coefficient for a linear uniformly elliptic partial differential equation in divergence form, in a bounded domain in dimension $d \leq 3$, from measurements of the solution in the interior. We adopt a Bayesian approach to the problem. We place a prior random field measure on the log permeability, specified through the Karhunen-Loève expansion of its draws. We consider Gaussian measures constructed this way, and study the regularity of functions drawn from them. We also study the Lipschitz properties of the observation operator mapping the log permeability to the observations. Combining these regularity and continuity estimates, we show that the posterior measure is well defined on a suitable Banach space. Furthermore the posterior measure is shown to be Lipschitz with respect to the data in the Hellinger metric, giving rise to a form of well posedness of the inverse problem. Determining the posterior measure, given the data, solves the problem of uncertainty quantification for this inverse problem. In practice the posterior measure must be approximated in a finite dimensional space. We quantify the errors incurred by employing a truncated Karhunen-Loève expansion to represent this meausure. In particular we study weak convergence of a general class of locally Lipschitz functions of the log permeability, and apply this general theory to estimate errors in the posterior mean of the pressure and the pressure covariance, under refinement of the finite-dimensional Karhunen-Loève truncation.
\end{abstract}

Key words. inverse problems, Bayesian approach, uncertainty quantification, elliptic inverse problem

AMS subject classifications. 35J99, 62G99

DOI. $10.1137 / 100814664$

1. Introduction. There is a growing interest in uncertainty quantification for differential equations in which the input data is uncertain. In the context of elliptic partial differential equations much of this work has concentrated on the problem of groundwater flow in which uncertainty enters the diffusion coefficient in a divergence form elliptic partial differential equation. Here there has been substantial work in the numerical analysis community devoted to quantifying the error in the solution of the problem in the case where the diffusion coefficient is a random field specified through a Karhunen-Loève or polynomial chaos expansion which is truncated $[2,3,4,7,6,16,20,21,23,24,25,29]$. However, in practice the unknown diffusion coefficient is often conditioned by observational data, leading to an inverse problem [22]. This gives rise to a far more complicated measure on the diffusion coefficient. The purpose of this paper is to study this inverse problem and, in particular, the effect of approximating the underlying probability measure via a finite, but large, set of real valued random variables. Much of the existing numerical analysis concerning groundwater flow with random permeability requires uniform upper and lower bounds over the probability space, and hence excludes the log normal permeability distributions widely used in applications. An exception is the recent paper [6] in which the effect of log normal diffusion coefficient on the pressure is studied, and weak approx-

${ }^{*}$ Received by the editors November 15, 2010; accepted for publication (in revised form) August 22, 2011; published electronically December 15, 2011.

http://www.siam.org/journals/sinum/49-6/81466.html

${ }^{\dagger}$ Mathematics Institute, University of Warwick, Coventry CV4 7AL, UK (M.Dashti@warwick. ac.uk, A.M.Stuart@warwick.ac.uk). The second author's work was supported by the EPSRC (UK) and ERC. 
imation quantified. For the inverse problem we study here we also use log normal priors; these are attractive from an inverse modeling perspective precisely because no prior bounds on the permeability may be known. A key tool when working with log normal distributions, and hence Gaussian measures, is the Fernique theorem which facilitates functional integration of a wide class of functions, including the exponential of quadratics, against Gaussian measures [12]. The paper [6] exemplifies the key role of the Fernique theorem and this theorem will also be used extensively in our developments of the inverse problem.

We consider the elliptic equation

$$
\begin{aligned}
-\nabla \cdot\left(\mathrm{e}^{u} \nabla p\right) & =f+\nabla \cdot g, \quad x \in D, \\
p & =\phi, \quad x \in \partial D,
\end{aligned}
$$

with $D$ an open, bounded, and connected subset of $\mathbb{R}^{d}, d \leq 3, p, u, f$, and $\phi$ scalar functions and $g$ a vector function on $D$. Given any $u \in L^{\infty}(D)$ we define $\lambda(u)$ and $\Lambda(u)$ by

$$
\lambda(u)=\operatorname{ess} \inf _{x \in D} \mathrm{e}^{u(x)}, \quad \Lambda(u)=\operatorname{ess} \sup _{x \in D} \mathrm{e}^{u(x)} .
$$

Where it causes no confusion we will simply write $\lambda$ or $\Lambda$. Equation (1.1) arises as a model for flow in a porous medium with $p$ the pressure (or the head) and $\mathrm{e}^{u}$ the permeability (or the transmissivity); the velocity $v$ is given by the formula $v \propto-\mathrm{e}^{u} \nabla p$.

Consider making noisy observations of a set of linear functionals $l_{j}$ of the pressure field $p$, so that $l_{j}: p \mapsto l_{j}(p) \in \mathbb{R}$. We write the observations as

$$
y_{j}=l_{j}(p)+\eta_{j}, \quad j=1, \ldots, K .
$$

We assume, for simplicity, that $\eta=\left\{\eta_{j}\right\}_{j=1}^{K}$ is a mean zero Gaussian observational noise with covariance $\Gamma$. In this paper we consider $l_{j}$ to be either

(a) pointwise evaluation of $p$ at a point $x_{j} \in D$ (assuming enough regularity for $f, g$ and $\phi$ so that this makes sense almost everywhere in $D$ ); or

(b) $l_{j}: H^{1}(D) \rightarrow \mathbb{R}$, a functional on $H^{1}(D)$ (again assuming enough regularity for $f, g$, and $\phi$ so that $\left.p \in H^{1}(D)\right)$.

Our objective is to determine $u$ from $y=\left\{y_{j}\right\}_{j=1}^{K} \in \mathbb{R}^{K}$. We adopt a probabilistic approach which we now outline. In the sequel we derive conditions under which we may view $l_{j}(p)$ as a function of $u$. Then, concatenating the data, we have

$$
y=\mathcal{G}(u)+\eta,
$$

with

$$
\mathcal{G}(u)=\left(l_{1}(p), \ldots, l_{\mathrm{K}}(p)\right)^{T} .
$$

Here the observation operator $\mathcal{G}$ maps $X$ into $\mathbb{R}^{K}$ where $X$ is a Banach space which we specify below in various scenarios and is determined by the forward model. From the properties of $\eta$ we see that the likelihood of the data $y$ given $u$ is

$$
\mathbb{P}(y \mid u) \propto \exp \left(-\frac{1}{2}\left|\Gamma^{-\frac{1}{2}}(y-\mathcal{G}(u))\right|^{2}\right),
$$

where $|\cdot|$ is the standard Euclidean norm. Let $\mathbb{P}(u)$ denote a prior distribution on the function $u$. If $u$ were finite dimensional, the posterior distribution, by Bayes' rule, would be given by

$$
\mathbb{P}(u \mid y) \propto \mathbb{P}(y \mid u) \mathbb{P}(u) .
$$


For infinite-dimensional spaces however, there is no density with respect to the Lebesgue measure. In this context Bayes rule should be interpreted as providing the RadonNikodym derivative between the posterior measure $\mu^{y}(\mathrm{~d} u)=\mathbb{P}(\mathrm{d} u \mid y)$ and the prior measure $\mu_{0}(\mathrm{~d} u)=\mathbb{P}(\mathrm{d} u)$ :

$$
\frac{d \mu^{y}}{d \mu_{0}}(u) \propto \exp \left(-\frac{1}{2}\left|\Gamma^{-\frac{1}{2}}(y-\mathcal{G}(u))\right|^{2}\right)
$$

The problem of making sense of Bayes rule for probability measures on function spaces with a Gaussian prior is addressed in $[8,9,27]$. In section 2 we recall these results, and then, in subsection 2.3 , we state and prove a new result concerning stability properties of the posterior measure with respect to finite-dimensional approximation of the prior.

In section 3 we show that the observation operator $\mathcal{G}$ of the elliptic problem described above satisfies boundedness and Lipschitz continuity conditions for appropriate choices of the Banach space $X$. In section 4 we combine the results of the preceding two sections to show that formula (1.5) holds for the posterior measure, and to study its approximation with respect to finite dimensional specification of the prior and posterior. Section 5 contains some concluding remarks.

2. Bayesian approach to inverse problems for functions. In this section we recall various theoretical results related to the development of Bayesian statistics on function space. We also state and prove a new result on the weak approximation of the posterior using finite-dimensional truncation of the Karhunen-Loève expansion. We assume that we are given two Banach spaces $X$ and $Y$, a function $\Phi: X \times Y \rightarrow \mathbb{R}$ and a probability measure $\mu_{0}$ supported on $X$. Consider the putative Radon-Nikodym derivative (derived by the conditioning of the joint measure on $(u, y) \in X \times Y$ on $y$, see [13])

$$
\begin{aligned}
\frac{d \mu^{y}}{d \mu_{0}}(u) & =\frac{1}{Z(y)} \exp (-\Phi(u ; y)) \\
Z(y) & =\int_{X} \exp (-\Phi(u ; y)) \mu_{0}(\mathrm{~d} u) .
\end{aligned}
$$

Our aim is to find conditions on $\Phi$ and $\mu_{0}$ under which $\mu^{y}$ is a well-defined probability measure on $X$, which is continuous in the data $y$, and to describe an approximation result for $\mu^{y}$ with respect to approximation of $\Phi$. Remarkably these results may all be proved simply by establishing properties of the operator $\Phi$ and its approximation on $X$, and then choosing the prior Gaussian measure so that $\mu_{0}(X)=1$. This clearly separates the analytic and probabilistic aspects of the Bayesian formulation of inverse problems for functions. The results of this section are independent of the specific inverse problem described in section 1 and have wide applicability. Note, however, that (1.5) is a particular case of the general set-up of (2.1), with $Y=\mathbb{R}^{K}$. But the level of generality we adopt allows us to work with infinite dimensional data (functions) and/or non-Gaussian observational error $\eta$. In particular if the data $y=\mathcal{G}(u)+\eta$, where $\mathcal{G}: X \rightarrow Y$ is the observation operator, $Y$ is a Hilbert space, and $\eta$ is a mean zero Gaussian random field on $Y$ with covariance operator $\Gamma$ and Cameron-Martin space $H(Y) \subset Y$ given by $\left(H(Y),\left\langle\Gamma^{-\frac{1}{2}} \cdot, \Gamma^{-\frac{1}{2}} \cdot\right\rangle_{Y},\left\|\Gamma^{-\frac{1}{2}} \cdot\right\|_{Y}\right)$, then we define $\Phi$ as

$$
\begin{aligned}
\Phi(u ; y) & =\frac{1}{2}\left\|\Gamma^{-\frac{1}{2}}(y-\mathcal{G}(u))\right\|_{Y}^{2}-\frac{1}{2}\left\|\Gamma^{-\frac{1}{2}} y\right\|_{Y}^{2} \\
& =\frac{1}{2}\left\|\Gamma^{-\frac{1}{2}} \mathcal{G}(u)\right\|_{Y}^{2}-\left\langle\Gamma^{-\frac{1}{2}} y, \Gamma^{-\frac{1}{2}} \mathcal{G}(u)\right\rangle_{Y} .
\end{aligned}
$$

Copyright (c) by SIAM. Unauthorized reproduction of this article is prohibited. 
On the other hand if $Y=\mathbb{R}^{K}$ and $\eta$ has Lebesgue density $\rho$, then we define $\Phi$ by the identity $\exp (-\Phi(u ; y))=\rho(y-\mathcal{G}(u))$. Note that these two definitions agree, up to an additive constant depending only on $y$, when $\eta$ is Gaussian and $Y$ is finite dimensional; such a constant simply amounts to adjusting the normalization $Z(y)$. The subtraction of the term $\frac{1}{2}\left\|\Gamma^{-\frac{1}{2}} y\right\|_{Y}^{2}$ in the infinite-dimensional data setting is required to make sure that $\Phi(\cdot ; y)$ is almost surely finite with respect to $\eta[27]$. For simplicity we work in the case where $X$ comprises periodic functions on the $d$-dimensional torus $\mathbb{T}^{d}$; generalizations are possible.

2.1. Well-defined and well-posed Bayesian inverse problems. In $[8,9,27]$, it is shown that some appropriate properties of the log likelihood $\Phi$ together with an appropriate choice of a Gaussian prior measure imply the existence of a well-posed Bayesian inverse problem. Here, we recall these results. To this end we assume the following conditions on $\Phi$.

Assumption 2.1. Let $X$ and $Y$ be Banach spaces. The function $\Phi: X \times Y \rightarrow \mathbb{R}$ satisfies

(i) for every $\epsilon>0$ and $r>0$ there is $M=M(\epsilon, r) \in \mathbb{R}$ such that for all $u \in X$, and for all $y \in Y$ such that $\|y\|_{Y}<r$,

$$
\Phi(u, y) \geq M-\epsilon\|u\|_{X}^{2} ;
$$

(ii) for every $r>0$ there exists $K=K(r)>0$ such that for all $u \in X, y \in Y$ with $\max \left\{\|u\|_{X},\|y\|_{Y}\right\}<r$

$$
\Phi(u, y) \leq K
$$

(iii) for every $r>0$ there exists $L=L(r)>0$ such that for all $u_{1}, u_{2} \in X$ and $y \in Y$ with $\max \left\{\left\|u_{1}\right\|_{X},\left\|u_{2}\right\|_{X},\|y\|_{Y}\right\}<r$

$$
\left|\Phi\left(u_{1}, y\right)-\Phi\left(u_{2}, y\right)\right| \leq L\left\|u_{1}-u_{2}\right\|_{X} ;
$$

(iv) for every $\epsilon>0$ and $r>0$, there is $C=C(\epsilon, r) \in \mathbb{R}$ such that for all $y_{1}, y_{2} \in Y$ with $\max \left\{\left\|y_{1}\right\|_{Y},\left\|y_{2}\right\|_{Y}\right\}<r$ and for every $u \in X$

$$
\left|\Phi\left(u, y_{1}\right)-\Phi\left(u, y_{2}\right)\right| \leq \exp \left(\epsilon\|u\|_{X}^{2}+C\right)\left\|y_{1}-y_{2}\right\|_{Y} .
$$

We now recall two results from [8] concerning well definedness and well posedness of the posterior measure.

Theorem 2.2 (see [8]). Let $\Phi$ satisfy Assumptions 2.1(i)-(iii). Assume that $\mu_{0}$ is a Gaussian measure with $\mu_{0}(X)=1$. Then $\mu^{y}$ given by $(2.1)$ is a well-defined probability measure.

One can also show continuity of the posterior in the Hellinger metric $d_{\text {Hell }}$ with respect to the data $y$. For any two measures $\mu$ and $\mu^{\prime}$ both absolutely continuous with respect to the same reference measure $\nu$, the Hellinger metric $d_{\text {Hell }}$ is defined by

$$
d_{\text {Hell }}\left(\mu, \mu^{\prime}\right)=\sqrt{\frac{1}{2} \int\left(\sqrt{\frac{\mathrm{d} \mu}{\mathrm{d} \nu}}-\sqrt{\frac{\mathrm{d} \mu^{\prime}}{\mathrm{d} \nu}}\right)^{2} d \nu} .
$$

The Hellinger metric is independent of the choice of reference measure $\nu$, the measure with respect to which both $\mu$ and $\mu^{\prime}$ are absolutely continuous. For function $G: X \rightarrow$ $S$ with $S$ a Banach space, we have

$$
\left\|\mathbb{E}^{\mu} G(u)-\mathbb{E}^{\mu^{\prime}} G(u)\right\|_{S} \leq C\left(\mathbb{E}^{\mu}\|G(u)\|_{S}^{2}+\mathbb{E}^{\mu^{\prime}}\|G(u)\|_{S}^{2}\right)^{\frac{1}{2}} d_{\mathrm{Hell}}\left(\mu, \mu^{\prime}\right) .
$$

Copyright (c) by SIAM. Unauthorized reproduction of this article is prohibited. 
In our elliptic inverse problem, for example, we will choose $S$ to be the space $H_{0}^{1}$ where the pressure lives, or $H_{0}^{1} \otimes H_{0}^{1}$, the natural space for pressure covariance. Theorem 2.3 which follows is hence quite useful: for example, it implies Lipschitz continuity of the posterior mean or posterior covariance with respect to data. (See [8, section 2]).

Theorem 2.3 (see [8]). Let $\Phi$ satisfy Assumptions 2.1(i)-(iv). Assume that $\mu_{0}$ is a Gaussian measure with $\mu_{0}(X)=1$. Then

$$
d_{\mathrm{Hell}}\left(\mu^{y}, \mu^{y^{\prime}}\right) \leq C\left\|y-y^{\prime}\right\|_{Y},
$$

where $C=C(r)$ with $\max \left\{\|y\|_{Y},\left\|y^{\prime}\right\|_{Y}\right\} \leq r$.

We note that under the assumptions of Theorem 2.3, one can also show that

$$
u^{*}=\arg \min _{u \in E}\left\{\Phi(u ; y)+\frac{1}{2}\|u\|_{E}^{2}\right\}
$$

with $\left(E,\|\cdot\|_{E}\right)$ the Cameron-Martin space of a centered Gaussian measure $\mu_{0}$, is well defined (see Theorem 5.2 of [27]). When $X$, and hence $E$, are finite dimensional it is readily seen from $(2.1 \mathrm{a})$ that the maximum a posterior estimator of $\mu^{y}$ equals $u^{*}$. This can be shown to be true for the infinite-dimensional case as well [14]. The function $u^{*}$ is the solution of the classical Tikhonov regularization of the inverse problem problem of finding $u$, given $\Phi(u ; y)$, when the regularization term is chosen to be $\frac{1}{2}\|u\|_{E}^{2}$. Thus there is a direct link between Bayesian regularization and classical regularization. However, a fully Bayesian approach, which would include probing the posterior distribution by, for example, Markov chain-Monte Carlo methods (MCMC), allows for the possibility of quantifying uncertainty. The conditions on $\Phi$ allow for the construction of MCMC methods in the infinite-dimensional setting, leading to methods which are robust under mesh refinement; thus we are following the approach of "sample then discretize," which is the Bayesian analogue of the optimization approach "optimize then discretize"; the advantages of adopting this approach are illustrated in [10] for an inverse problem arising in fluid mechanics and discussed in greater generality in [11]. In addition, a discussion of the relationship between the classical and Bayesian approach to inverse problems and the numerical algorithms used in each context, can be found in [27, section 5].

2.2. Approximation of the posterior. In this section we recall a result concerning approximation of $\mu^{y}$ on the Banach space $X$ when the function $\Phi$ is approximated. This will be used in the next subsection for approximation of $\mu^{y}$ on a finite-dimensional space. Consider $\Phi^{N}$ to be an approximation of $\Phi$. Here we state a result which quantifies the effect of this approximation in the posterior measure in terms of the approximation error in $\Phi$.

Define $\mu^{y, N}$ by

$$
\begin{aligned}
\frac{\mathrm{d} \mu^{y, N}}{\mathrm{~d} \mu_{0}}(u) & =\frac{1}{Z^{N}(y)} \exp \left(-\Phi^{N}(u)\right), \\
Z^{N}(y) & =\int_{X} \exp \left(-\Phi^{N}(u)\right) \mathrm{d} \mu_{0}(u) .
\end{aligned}
$$

We suppress the dependence of $\Phi$ and $\Phi^{N}$ on $y$ in this section as it is considered fixed.

THEOREM 2.4 (see [9]). Assume that the measures $\mu$ and $\mu^{N}$ are both absolutely continuous with respect to a Gaussian $\mu_{0}$ with $\mu_{0}(X)=1$, and given by (2.1) and (2.3), respectively. Suppose that $\Phi$ and $\Phi^{N}$ satisfy Assumptions 2.1(i) and (ii), uniformly in 
$N$, and that for any $\epsilon>0$ there exists $C=C(\epsilon) \in \mathbb{R}$ such that

$$
\left|\Phi(u)-\Phi^{N}(u)\right| \leq \exp \left(\epsilon\|u\|_{X}^{2}+C\right) \psi(N),
$$

where $\psi(N) \rightarrow 0$ as $N \rightarrow \infty$. Then there exists a constant independent of $N$ such that

$$
d_{\text {Hell }}\left(\mu, \mu^{N}\right) \leq C \psi(N) .
$$

2.3. Approximating the posterior measure in a finite-dimensional space. In this section, we again consider approximation of the posterior measure for the inverse problem on $X \subseteq L^{2}\left(\mathbb{T}^{d}\right)$ where $\mathbb{T}$ denotes the unit cube $[-\pi, \pi)^{d}$ with opposite faces identified. But here we additionally assume that the approximation is made in a finite-dimensional subspace and hence corresponds to something that can be implemented computationally. Our approximation space will be defined by truncating the Karhunen-Loève basis $\left\{\varphi_{l}\right\}_{l=1}^{\infty}$ comprising the eigenfunctions of the covariance operator of the Gaussian measure $\mu_{0}$. For simplicity we assume from now on that $\mu_{0}$ is centered (zero mean).

Define the subspace $W^{N}$ spanned by the $\left\{\varphi_{l}\right\}_{l=1}^{N}$ and let $W^{\perp}$ denote the complement of $W^{N}$ in $L^{2}\left(\mathbb{T}^{d}\right)$. For any $u \in X$ let

$$
u^{N}=\sum_{l=1}^{N} u_{l} \varphi_{l}, \quad \text { with } u_{l}=\left(u, \varphi_{l}\right),
$$

where $(\cdot, \cdot)$ is the $L^{2}$-inner product. The approximate posterior measure will induce a measure on the coefficients $\left\{u_{l}\right\}_{l=1}^{N}$ appearing in (2.4), and hence a measure $\nu^{N}$ on $u^{N} \in W^{N}$. Our interest is in quantifying the error incurred when approximating expectations under $\mu^{y}$ by expectations under $\nu^{N}$.

For simplicity we consider the case where the data $y$ is finite dimensional and the posterior measure is defined via (2.1) with

$$
\Phi(u):=\frac{1}{2}\left|\Gamma^{-\frac{1}{2}}(y-\mathcal{G}(u))\right|^{2} .
$$

Here $|\cdot|$ denotes the Euclidean norm and $\Gamma$ is assumed positive and symmetric. We drop explicit $y$ dependence in $\Phi$ (and approximation $\Phi^{N}$ ) throughout this section.

Note that $\mu_{0}$ factors as the product of two independent measures $\mu_{0}^{N} \otimes \mu_{0}^{\perp}$ on $W^{N} \oplus W^{\perp}$. Let $P^{N}$ be the orthogonal projection of $L^{2}\left(\mathbb{T}^{d}\right)$ onto $W^{N}$, and $P^{\perp}=$ $I-P^{N}$. Any $u \in X$ can be written as $u=u^{N}+u^{\perp}$, where $u^{N}=P^{N} u$ is given by (2.4) and $u^{\perp}=P^{\perp} u$. We define $\mathcal{G}^{N}(\cdot)=\mathcal{G}\left(P^{N}\right.$.) and consider the approximate measure (2.3) with $\Phi^{N}$ given by

$$
\Phi^{N}(u)=\frac{1}{2}\left|\Gamma^{-1 / 2}\left(y-\mathcal{G}^{N}(u)\right)\right|^{2} .
$$

Because $\mathcal{G}^{N}(u)$ depends only on $u^{N}$, and because $\mu_{0}=\mu_{0}^{N} \otimes \mu_{0}^{\perp}$ the resulting measure $\mu^{y, N}$ on $X$ can be factored as $\mu^{y, N}=\nu^{N} \otimes \mu^{\perp}$, where

$$
\frac{\mathrm{d} \nu^{N}}{\mathrm{~d} \mu_{0}^{N}}(u) \propto \exp \left(-\frac{1}{2}\left|\Gamma^{-1 / 2}\left(y-\mathcal{G}^{N}(u)\right)\right|^{2}\right)
$$

and $\mu^{\perp}=\mu_{0}^{\perp}$. The measure $\nu^{N}$ given by (2.7) is finite dimensional and amenable to statistical sampling techniques, such as MCMC. For the purposes of this paper 
we assume that expectations with respect to $\nu^{N}$ on $W^{N}$ can be computed exactly. Discussion of MCMC methods which are robust to increasing $N$, can be found at the end of subsection 2.1 .

We are interested in approximating expectations under $\mu^{y}$ of functions $G: X \rightarrow S$, $S$ a Banach space. For example, $G(u)$ may denote the pressure field, or covariance of the pressure field for the elliptic inverse in section 1. Abusing notation, we will sometimes write $G(u)=G\left(u^{N}, u^{\perp}\right)$. In practice we are able to compute expectations of $G\left(u^{N}, 0\right)$ under $\nu^{N}$. Thus we are interested in estimating the weak error

$$
e=\left\|\mathbb{E}^{\mu^{y}} G\left(u^{N}, u^{\perp}\right)-\mathbb{E}^{\nu^{N}} G\left(u^{N}, 0\right)\right\|_{S} .
$$

We now state and prove a theorem concerning this error, under the following assumptions on $\mathcal{G}$ and $G$.

Assumption 2.5. Assume that $X, X^{\prime}$, and $X^{\prime \prime}$ are Banach spaces, and $X$ is continuously embedded into $X^{\prime}$ and $X^{\prime}$ is continuously embedded into $X^{\prime \prime}$. Suppose also that the centered Gaussian probability measure $\mu_{0}$ satisfies $\mu_{0}(X)=1$. Then, for all $\epsilon>0$, there is $K=K(\epsilon) \in(0, \infty)$, such that, for all $u_{1}, u_{2} \in X$,

$$
\begin{aligned}
\left|\mathcal{G}\left(u_{1}\right)-\mathcal{G}\left(u_{2}\right)\right| & \leq K \exp \left(\epsilon \max \left\{\left\|u_{1}\right\|_{X}^{2},\left\|u_{2}\right\|_{X^{\prime}}^{2}\right\}\right)\left\|u_{1}-u_{2}\right\|_{X^{\prime \prime}}, \\
\left\|G\left(u_{1}\right)-G\left(u_{2}\right)\right\|_{S} & \leq K \exp \left(\epsilon \max \left\{\left\|u_{1}\right\|_{X}^{2},\left\|u_{2}\right\|_{X^{\prime}}^{2}\right\}\right)\left\|u_{1}-u_{2}\right\|_{X^{\prime \prime}}
\end{aligned}
$$

Furthermore, $\left\|P^{\perp}\right\|_{\mathcal{L}\left(X, X^{\prime \prime}\right)}=\psi(N) \rightarrow 0$ as $N \rightarrow \infty$, and $\left\|P^{\perp}\right\|_{\mathcal{L}\left(X, X^{\prime}\right)}$ is bounded independently of $N$.

TheOREM 2.6. Let Assumption 2.5 hold and assume that $\Phi$ and $\Phi^{N}$ are given by (2.5) and (2.6), respectively. Then the probability measures $\mu^{y}$ and $\mu^{y, N}$ are absolutely continuous with respect to $\mu_{0}$ and given by (2.1) and (2.3). Furthermore, the weak error (2.8) satisfies e $\leq C \psi(N)$ as $N \rightarrow \infty$.

Proof. Since $X \hookrightarrow X^{\prime} \hookrightarrow X^{\prime \prime}$ we have, for some constants $C_{1}, C_{2}>0$, \| . $\left\|_{X^{\prime \prime}} \leq C_{1}\right\| \cdot\left\|_{X^{\prime}} \leq C_{2}\right\| \cdot \|_{X}$. From the assumptions on $\mathcal{G}$ and $P^{\perp}$ we deduce that Assumptions 2.1(i)-(iii) hold for $\Phi$ and $\Phi^{N}$ given by (2.5) and (2.6), respectively. Thus $\mu^{y}$ and $\mu^{y, N}$ are well-defined probability measures, both absolutely continuous with respect to $\mu_{0}$, and satisfying $\mu^{y}(X)=\mu^{y, N}(X)=1$. By the triangle inequality we have $e \leq e_{1}+e_{2}$ where

$$
\begin{aligned}
& e_{1}=\left\|\mathbb{E}^{\mu^{y}} G\left(u^{N}, u^{\perp}\right)-\mathbb{E}^{\mu^{y, N}} G\left(u^{N}, u^{\perp}\right)\right\|_{S}, \\
& e_{2}=\left\|\mathbb{E}^{\mu^{y, N}} G\left(u^{N}, u^{\perp}\right)-\mathbb{E}^{\nu^{N}} G\left(u^{N}, 0\right)\right\|_{S} .
\end{aligned}
$$

We first estimate $e_{1}$. Note that, by use of the assumptions on $\mathcal{G}$ and $\mathcal{G}^{N}$, we have that for any $\epsilon>0$ there is $K=K(\epsilon) \in(0, \infty)$ such that

$$
\begin{aligned}
\left|\Phi(u)-\Phi^{N}(u)\right| & \leq \frac{1}{2}\left|\Gamma^{-\frac{1}{2}}\left(2 y-\mathcal{G}(u)-\mathcal{G}^{N}(u)\right)\right|\left|\Gamma^{-\frac{1}{2}}\left(\mathcal{G}(u)-\mathcal{G}^{N}(u)\right)\right| \\
& \leq K \exp \left(\epsilon\|u\|_{X}^{2}\right) \psi(N) .
\end{aligned}
$$

By Theorem 2.4 we deduce that the Hellinger distance between $\mu^{y}$ and $\mu^{y, N}$ tends to zero like $\psi(N)$ and hence, by $(2.2)$, that $e_{1} \leq C \psi(N)$. This last bound follows after noting that the required integrability of $\|G(u)\|_{S}^{2}$ and $\left\|G\left(u^{N}\right)\right\|_{S}^{2}$ follows from the Lipschitz bound on $G$, the operator norm bound on $P^{\perp}$ and the Fernique theorem [12].

We now estimate $e_{2}$. Because $\mathbb{E}^{\mu^{y, N}} G\left(u^{N}, 0\right)=\mathbb{E}^{\nu^{N}} G\left(u^{N}, 0\right)$ we obtain

$$
e_{2} \leq \mathbb{E}^{\mu^{y, N}}\left\|G(u)-G\left(u^{N}\right)\right\|_{S}
$$

Copyright $@$ by SIAM. Unauthorized reproduction of this article is prohibited. 
From the Lipschtiz properties of $G$ we deduce that

$$
\begin{aligned}
e_{2} & \leq K_{1}(\epsilon) \mathbb{E}^{\mu^{y, N}}\left(\exp \left(\epsilon \max \left\{\|u\|_{X}^{2},\left\|P^{N} u\right\|_{X^{\prime}}^{2}\right\}\right)\left\|P^{\perp} u\right\|_{X^{\prime \prime}}\right) \\
& \leq K_{2}(\epsilon) \mathbb{E}^{\mu^{y, N}}\left(\exp \left(C \epsilon\|u\|_{X}^{2}\right)\|u\|_{X}\right) \psi(N) \\
& \leq K_{3}(\epsilon) \mathbb{E}^{\mu^{y, N}}\left(\exp \left(2 C \epsilon\|u\|_{X}^{2}\right)\right) \psi(N),
\end{aligned}
$$

where $C$ is independent of $\epsilon$. The result follows by the Fernique theorem.

The results of this section are quite general, concerning a wide class of Bayesian inverse problems for functions, using Gaussian priors. These results can also be generalized to the case of Besov priors introduced in [18] and this approach is pursued in [13]. In the next section we establish conditions which enable application of Theorems $2.2,2.3,2.4$, and 2.6 to the specific elliptic inverse problem described in section 1 . We then apply these results in section 4 .

3. Estimates on the observation operator. In order to apply Theorems 2.2, $2.3,2.4$, and 2.6 to the elliptic inverse problem described in section 1 we need to prove certain properties of the observation operator $\mathcal{G}$ given by (1.4), viewed as a mapping from a Banach space $X$ into $\mathbb{R}^{m}$, and the function $G: X \rightarrow S$. Then the prior measure $\mu_{0}$ must be chosen so that $\mu_{0}(X)=1$. It is hence desirable to find spaces $X$ which are as large as possible, so as not to unduly restrict the prior, but for which the desired properties of the observation operator hold. As discussed in section 1 we consider the observation operator obtained from either the pointwise measurements of $p$ or bounded linear functionals of $p \in H^{1}(D)$. We obtain the bounds on the observation operator for each of these cases in the following.

3.1. Measurements from bounded linear functionals of $p \in H^{1}(D)$. In this case, using standard energy estimates, we have the following result.

Proposition 3.1. Consider (1.1) with $D \subset \mathbb{R}^{d}, d=2,3$, a bounded domain, the boundary of $D, \partial D, C^{1}$-regular, $f \in H^{-1}(D), g \in L^{2}(D)$, and assume that $\phi$ may be extended to a function $\phi \in H^{1}(D)$. Then there exists a constant $C=$ $C\left(\|f\|_{H^{-1}},\|g\|_{L^{2}},\|\phi\|_{H^{1}(D)}\right)$ such that

(i) if $u \in L^{\infty}(D)$, then

$$
\|p\|_{H^{1}(D)} \leq C \exp \left(2\|u\|_{L^{\infty}(D)}\right)
$$

(ii) if $u_{1}, u_{2} \in L^{\infty}(D)$, and $p_{1}, p_{2}$ are the corresponding solutions of (1.1), then

$$
\left\|p_{1}-p_{2}\right\|_{H^{1}(D)} \leq C \exp \left(4 \max \left\{\left\|u_{1}\right\|_{L^{\infty}},\left\|u_{2}\right\|_{L^{\infty}}\right\}\right)\left\|u_{1}-u_{2}\right\|_{L^{\infty}} .
$$

Corollary 3.2. Let the assumptions of Proposition 3.1 hold. Consider $\mathcal{G}(u)=$ $\left(l_{1}(p), \ldots, l_{\mathrm{K}}(p)\right)$ with $l_{j}, j=1, \ldots, K$, bounded linear functionals on $H^{1}(D)$. Then for any $u, u_{1}, u_{2} \in L^{\infty}(D)$ we have

$$
|\mathcal{G}(u)| \leq C \exp \left(2\|u\|_{L^{\infty}(D)}\right)
$$

and

$$
\left|\mathcal{G}\left(u_{1}\right)-\mathcal{G}\left(u_{2}\right)\right| \leq C \exp \left(4 \max \left\{\left\|u_{1}\right\|_{L^{\infty}},\left\|u_{2}\right\|_{L^{\infty}}\right\}\right)\left\|u_{1}-u_{2}\right\|_{L^{\infty}(D)},
$$

with $C=C\left(K,\|f\|_{H^{-1}},\|g\|_{L^{2}},\|\phi\|_{H^{1}(D)}, \max _{j}\left\|l_{j}\right\|_{H^{-1}}\right)$. 
Proof of Proposition 3.1. (i) Substituting $q=p-\phi$ in (1.1) and taking the inner product with $q$, we obtain

$$
\mathrm{e}^{-\|u\|_{L^{\infty}}}\|\nabla q\|_{L^{2}} \leq \mathrm{e}^{\|u\|_{L^{\infty}}}\|\nabla \phi\|_{L^{2}}+\|f\|_{H^{-1}}+\|g\|_{L^{2}}
$$

and therefore

$$
\|\nabla p\|_{L^{2}} \leq\left(1+\mathrm{e}^{2\|u\|_{L^{\infty}}}\right)\|\nabla \phi\|_{L^{2}}+\mathrm{e}^{\|u\|_{L^{\infty}}}\left(\|f\|_{H^{-1}}+\|g\|_{L^{2}}\right),
$$

which implies the result of part (i).

(ii) The difference $p_{1}-p_{2}$ satisfies

$$
\nabla \cdot\left(\mathrm{e}^{u_{1}} \nabla\left(p_{1}-p_{2}\right)\right)=\nabla \cdot\left(\left(\mathrm{e}^{u_{2}}-\mathrm{e}^{u_{1}}\right) \nabla p_{2}\right) .
$$

Taking the inner product of the equation with $p_{1}-p_{2}$ gives

$$
\mathrm{e}^{-\left\|u_{1}\right\|_{L^{\infty}}}\left\|\nabla\left(p_{1}-p_{2}\right)\right\|_{L^{2}}^{2} \leq\left\|e^{u_{2}}-e^{u_{1}}\right\|_{L^{\infty}}\left\|\nabla p_{2}\right\|_{L^{2}}\left\|\nabla\left(p_{1}-p_{2}\right)\right\|_{L^{2}} .
$$

For any $x \in D$ we can write

$$
\begin{aligned}
\left|\mathrm{e}^{u_{2}(x)}-\mathrm{e}^{u_{1}(x)}\right| & =\left|\left(u_{2}(x)-u_{1}(x)\right) \int_{0}^{1} \mathrm{e}^{s u_{2}+(1-s) u_{1}} \mathrm{~d} s\right| \\
& \leq\left\|u_{2}-u_{1}\right\|_{L^{\infty}} \mathrm{e}^{\max \left\{\left\|u_{1}\right\|_{L} \infty,\left\|u_{2}\right\|_{L^{\infty}}\right\}} .
\end{aligned}
$$

Hence using the estimate for $\left\|\nabla p_{2}\right\|_{L^{2}}$ from part (i), the result follows.

3.2. Pointwise measurements of $\boldsymbol{p}$. Here we obtain bounds on the $L^{\infty}$-norm of the pressure $p$.

Proposition 3.3. Consider (1.1) with $D \subset \mathbb{R}^{d}, d=2,3$, a bounded domain, the boundary of $D, \partial D, C^{1}$-regular, $f \in L^{r}(D), g \in L^{2 r}(D)$ with $r>d / 2$, and $\phi \in L^{\infty}(\partial D)$. There exists $C=C\left(K, d, r, D, \sup _{\partial D}|\phi|,\|f\|_{L^{r}},\|g\|_{L^{2 r}}\right)$ such that

$$
\|p\|_{L^{\infty}(D)} \leq C \exp \left(\|u\|_{L^{\infty}(D)}\right) .
$$

Proposition 3.4. Let the assumptions of Proposition 3.3 hold. Suppose also that $\phi$ may be extended to a function $\phi \in W^{1,2 r}(D)$. Then

(i) the mapping $u \mapsto p$ from $C(\bar{D})$ into $L^{\infty}(D)$ is locally Lipschitz continuous;

(ii) assume that $u_{1} \in L^{\infty}(D), u_{2} \in C^{t}(D)$ for some $t>0$, and $p_{1}, p_{2}$ are the corresponding solutions of (1.1). Then for any $\epsilon>0$ there exists $C$ depending on $K, d, t, \epsilon,\|f\|_{L^{r}},\|g\|_{L^{2 r}}$, and $\|\phi\|_{W^{1,2 r}}$ such that

$$
\left\|p_{1}-p_{2}\right\|_{L^{\infty}(D)} \leq C \exp \left(c_{0} \max \left\{\left\|u_{1}\right\|_{L^{\infty}(D)},\left\|u_{2}\right\|_{C^{t}(D)}\right\}\right)\left\|u_{1}-u_{2}\right\|_{L^{\infty}(D)},
$$

where $c_{0}=4+(4+2 d) / t+\epsilon$.

Corollary 3.5. Let the assumptions of Proposition 3.3 hold. Consider $\mathcal{G}(u)=$ $\left(l_{1}(p), \ldots, l_{\mathrm{K}}(p)\right)$ with $l_{j}, j=1, \ldots, K$, pointwise evaluations of $p$ at $x_{j} \in D$. Then for any $u \in L^{\infty}(D)$ there exists $C=C\left(K, d, r, D\right.$, $\left.\sup _{\partial D}|\phi|,\|f\|_{L^{r}},\|g\|_{L^{2 r}}\right)$ such that

$$
|\mathcal{G}(u)| \leq C \exp \left(\|u\|_{L^{\infty}(D)}\right) .
$$

If $u_{1} \in L^{\infty}(D), u_{2} \in C^{t}(D)$ for some $t>0$ and $\phi$ can be extended to a function $\phi \in W^{1,2 r}(D)$, then, for any $\epsilon>0$,

$$
\left|\mathcal{G}\left(u_{1}\right)-\mathcal{G}\left(u_{2}\right)\right| \leq C \exp \left(c_{0} \max \left\{\left\|u_{1}\right\|_{L^{\infty}(D)},\left\|u_{2}\right\|_{C^{t}(D)}\right\}\right)\left\|u_{1}-u_{2}\right\|_{L^{\infty}(D)}
$$

with $C=C\left(K, d, t, \epsilon,\|f\|_{L^{r}},\|g\|_{L^{2 r}},\|\phi\|_{W^{1,2 r}}\right)$ and $c_{0}=4+(4+2 d) / t+\epsilon$ for any $\epsilon>0$.

Copyright (c) by SIAM. Unauthorized reproduction of this article is prohibited. 
Proof of Proposition 3.3. By Theorems 8.15 and 8.17 of [17] we have, recalling the definition of $\lambda$ in (1.2),

$$
\sup _{D}|p| \leq \sup _{\partial D}|\phi|+C(d, r,|D|)\left(\left\|p_{0}\right\|_{L^{2}}+\frac{1}{\lambda}\|f\|_{L^{r}}+\frac{1}{\lambda}\|g\|_{L^{2 r}}\right),
$$

where $p_{0}$ is the solution of (1.1) with $\phi=0$. Taking the inner product of (1.1) with $p_{0}$ we obtain, for $c_{i}=c_{i}(d, r,|D|)$,

$$
\begin{aligned}
\left\|\nabla p_{0}\right\|_{L^{2}} & \leq \frac{c_{1}}{\lambda}\left(\|f\|_{H^{-1}}+\|g\|_{L^{2}}\right) \\
& \leq \frac{c_{2}}{\lambda}\left(\|f\|_{L^{r}}+\|g\|_{L^{2}}\right) .
\end{aligned}
$$

Hence

$$
\sup _{D}|p| \leq \frac{1}{\lambda} C(d, r, D)\left(\sup _{\partial D}|\phi|+\|f\|_{L^{r}}+\|g\|_{L^{2 r}}\right)
$$

and the result follows since $\lambda \geq \mathrm{e}^{-\|u\|_{L}(D)}$.

Proof of Proposition 3.4. The difference $p_{1}-p_{2}$ satisfies

$$
\begin{aligned}
\nabla \cdot\left(\mathrm{e}^{u_{1}} \nabla\left(p_{1}-p_{2}\right)\right) & =\nabla \cdot F, \quad x \in D, \\
p_{1}(x) & =p_{2}(x), \quad x \in \partial D,
\end{aligned}
$$

where

$$
F=-\left(e^{u_{1}(x)}-e^{u_{2}(x)}\right) \nabla p_{2}
$$

Let

$$
\lambda_{m}=\min \left\{\lambda\left(u_{1}\right), \lambda\left(u_{2}\right)\right\} \quad \text { and } \quad \Lambda_{m}=\max \left\{\Lambda\left(u_{1}\right), \Lambda\left(u_{2}\right)\right\} .
$$

By (3.2) we have

$$
\sup _{D}\left|p_{1}-p_{2}\right| \leq \frac{C(d, r, D)}{\lambda_{m}}\|F\|_{L^{2 r}}
$$

for $r>d / 2$. Now, $\|F\|_{L^{2 r}}$ may be estimated as follows, using Theorem A.3,

$$
\begin{aligned}
\|F\|_{L^{2 r}} & \leq\left\|\left(\mathrm{e}^{u_{1}}-\mathrm{e}^{u_{2}}\right) \nabla p_{2}\right\|_{L^{2 r}} \\
& \leq \Lambda_{m}\left\|u_{1}-u_{2}\right\|_{L^{\infty}}\left\|\nabla p_{2}\right\|_{L^{2 r}} \\
& \leq \Lambda_{m} C\left(d, r, D, u_{2}\right)\left(\|f\|_{L^{r}}+\|g\|_{L^{2 r}}+\|\phi\|_{W^{1,2 r}}\right)\left\|u_{1}-u_{2}\right\|_{L^{\infty}(D)}
\end{aligned}
$$

where in the last line we have used the fact that $2 r d /(2 r+d)<r$. This gives the Lipschitz continuity.

To show the second part, we use (3.4) and Corollary A.4 to write

$$
\begin{aligned}
\left\|p_{1}-p_{2}\right\|_{L^{\infty}(D)} \leq & \frac{1}{\lambda_{m}} C(d, r, D)\|F\|_{L^{2 r}} \\
\leq & \frac{\Lambda_{m}}{\lambda_{m}} C(d, r, D)\left\|\nabla p_{2}\right\|_{L^{2 r}}\left\|u_{1}-u_{2}\right\|_{L^{\infty}(D)} \\
\leq & C(d, D, r, t) \frac{\Lambda_{m}\left(1+\Lambda_{m}\right)}{\lambda_{m}^{2}}\left(1+\left(\Lambda_{m} / \lambda_{m}\right)^{\frac{1+d}{t}}\left\|u_{2}\right\|_{C^{t}(D)}^{\frac{1+d}{t}}\right) \\
& \times\left(1+\left(\Lambda_{m} / \lambda_{m}\right)^{\frac{1}{t}}\left\|u_{2}\right\|_{C^{t}(D)}^{\frac{1}{t}}\right) \\
& \times\left(\|f\|_{L^{r}}+\|g\|_{L^{2 r}}+\|\phi\|_{W^{1,2 r}}\right)\left\|u_{1}-u_{2}\right\|_{L^{\infty}(D)} .
\end{aligned}
$$

Copyright (c) by SIAM. Unauthorized reproduction of this article is prohibited. 
Since $1 / \lambda_{m}, \Lambda_{m} \leq \exp \left\{\left\|u_{1}\right\|_{C^{t}},\left\|u_{2}\right\|_{C^{t}}\right\}$ and for any $\epsilon>0$ there exists $C(\epsilon)$ such that

$$
\begin{aligned}
& \frac{\Lambda_{m}\left(1+\Lambda_{m}\right)}{\lambda_{m}^{2}}\left(1+\left(\Lambda_{m} / \lambda_{m}\right)^{\frac{1+d}{t}}\left\|u_{2}\right\|_{C^{t}(D)}^{\frac{1+d}{t}}\right)\left(1+\left(\Lambda_{m} / \lambda_{m}\right)^{\frac{1}{t}}\left\|u_{2}\right\|_{C^{t}(D)}^{\frac{1}{t}}\right) \\
& \quad \leq C(\epsilon) \exp \left(c_{0}(t) \max \left\{\left\|u_{1}\right\|_{L^{\infty}},\left\|u_{2}\right\|_{C^{t}}\right\}\right)
\end{aligned}
$$

with $c_{0}=4+(4+2 d) / t+\epsilon$. The result follows.

We can now summarize our assumptions on the forcing function and boundary conditions of (1.1) for our two choices of the observation operator $\mathcal{G}$ as follows. We will use these assumptions in subsequent sections. In both cases covered by these assumptions it is a consequence that $\Phi$ and $\mathcal{G}$ satisfy Assumptions 2.1 and 2.5.

Assumption 3.6. We consider the observation operator $\mathcal{G}(u)=\left(l_{1}(p), \ldots, l_{\mathrm{K}}(p)\right)$ defined as in (1.4) with $D \subset \mathbb{R}^{d}$ a bounded open set with $C^{1}$ regular boundary. Then either

(i) the mapping $l_{j}$ is a bounded linear functional on $H^{1}(D)$ for $j=1, \ldots, K$ and we assume that $f \in H^{-1}(D), g \in L^{2}(D)$, and $\phi$ may be extended to $\phi \in H^{1}(D)$; or

(ii) the mapping $l_{j}$ is the pointwise evaluation of $p$ at a point $x_{j} \in D$ for $j=$ $1, \ldots, K$ and we assume that $f \in L^{r}(D), g \in L^{2 r}(D)$, and $\phi$ may be extended to $\phi \in W^{1,2 r}(D)$, with $r>d / 2$.

4. Properties of the posterior measure for the elliptic inverse problem. We use the properties of the observation operator $\mathcal{G}$ for the elliptic problem to establish well posedness of the inverse problem, and to study approximation of the posterior measure in finite-dimensional spaces defined via Fourier truncation.

4.1. Well posedness of the posterior measure. Here we show the well definedness of the posterior measure and its continuity with respect to the data for the elliptic problem. We have the following theorem.

THEOREM 4.1. Consider the inverse problem for finding u from noisy observations of $p$ in the form of (1.3) and with $p$ solving (1.1) in $D=(-\pi, \pi)^{d}$. Let Assumption 3.6 hold and consider $\mu_{0}$ to be distributed as $\mathcal{N}\left(0,(-\Delta)^{-s}\right)$ with $\Delta$ the Laplacian operator acting on $H^{2}\left(\mathbb{T}^{d}\right)$ Sobolev functions with zero average on $\mathbb{T}^{d}$, and $s>d / 2$. Then the measure $\mu(\mathrm{d} u \mid y)=\mathbb{P}(\mathrm{d} u \mid y)$ is absolutely continuous with respect to $\mu_{0}$ with Radon-Nikodym derivative given by (2.1), (2.5). Furthermore, the posterior measure is continuous in the Hellinger metric with respect to the data:

$$
d_{\mathrm{Hell}}\left(\mu^{y}, \mu^{y^{\prime}}\right) \leq C\left|y-y^{\prime}\right| .
$$

Proof. Let $X=C^{t}(D)$. We first define measures $\pi_{0}$ and $\pi$ on $X \times \mathbb{R}^{K}$ as follows. Define $\pi_{0}(\mathrm{~d} u, \mathrm{~d} y)=\mu_{0}(\mathrm{~d} u) \otimes \rho(\mathrm{d} y)$, where $\rho$ is a centered Gaussian with covariance matrix $\Gamma$, assumed positive. Since $\mathcal{G}: X \rightarrow \mathbb{R}^{K}$ is continuous and since Lemma 6.25 of [27] shows that $\mu_{0}(X)=1$ for any $t \in(0, s-d / 2)$, we deduce that $\mathcal{G}$ is $\mu_{0}$ measurable for $t \in(0, s-d / 2)$. We define $\rho(\mathrm{d} y \mid u)=\mathcal{N}(\mathcal{G}(u), \Gamma)$ and then by $\mu_{0}$-measurability of $\mathcal{G}$, $\pi(\mathrm{d} u, \mathrm{~d} y)=\rho(\mathrm{d} y \mid u) \mu_{0}(\mathrm{~d} u)$. From the properties of Gaussian measure on $\mathbb{R}^{K}$ we have

$$
\frac{\mathrm{d} \pi}{\mathrm{d} \pi_{0}}(u, y) \propto \exp (-\Phi(u ; y))
$$

with $\Phi(u ; y)=\frac{1}{2}\left|\Gamma^{-1 / 2}(y-\mathcal{G}(u))\right|^{2}$. We now show that Assumptions 2.1(i)-(iv) hold for this $\Phi$. Assumption 2.1(i) is automatic because

$$
\Phi(u ; y):=\frac{1}{2}\left|\Gamma^{-\frac{1}{2}}(y-\mathcal{G}(u))\right|^{2} \geq 0 .
$$

Copyright $@$ by SIAM. Unauthorized reproduction of this article is prohibited. 
By Corollaries 3.2 and $3.5, \mathcal{G}$ is bounded on bounded sets in $X$, and Lipschitz on $X$, for any $t>0$, proving Assumptions 2.1(ii), (iii). To prove Assumption 2.1(iv) note that

$$
\begin{aligned}
\left|\Phi\left(u ; y_{1}\right)-\Phi\left(u ; y_{2}\right)\right| & \leq \frac{1}{2}\left|\Gamma^{-\frac{1}{2}}\left(y_{1}+y_{2}-\mathcal{G}(u)\right)\right|\left|\Gamma^{-\frac{1}{2}}\left(y_{1}-y_{2}\right)\right| \\
& \leq c_{1} \exp \left(c_{2}\|u\|_{X}\right)\left|y_{1}-y_{2}\right|
\end{aligned}
$$

and

$$
\exp \left(c\|u\|_{X}\right) \leq c_{1}(\epsilon) \exp \left(\epsilon\|u\|_{X}^{2}\right)
$$

By Assumption 2.1(ii) we deduce that

$$
\int_{X} \exp (-\Phi(u ; y)) \mu_{0}(\mathrm{~d} y) \geq \exp (-K(r)) \mu_{0}\left(\|u\|_{X}<r\right)>0
$$

By conditioning, as in Theorem 6.31 of [27], we deduce that the regular conditional probability $\mu^{y}(\mathrm{~d} u)=\mathbb{P}^{\pi}(\mathrm{d} u \mid y)$ is absolutely continuous with respect to $\mu_{0}$ and is given by (1.5). Continuity in the Hellinger metric follows from Theorem 2.3.

In the case that the spatial domain $D \subset \mathbb{R}^{n}$ is open and bounded with Lipschitz boundary, the prior measure $\mu_{0} \sim \mathcal{N}\left(0, \mathcal{A}^{-s}\right)$ with $s>d-1 / 2$ and $\mathcal{A}$ is the Laplacian operator acting on $D(\mathcal{A})=\left\{u \in H^{2}(D):\left.\nabla u \cdot \mathbf{n}\right|_{\partial D}=0\right.$ and $\left.\int_{D} u=0\right\}$ satisfies $\mu_{0}\left(C^{t}(D)\right)=1, t<s-d+1 / 2$. Hence the techniques of the preceding theorem can be used to show well posedness of the posterior in this case provided $s>d-\frac{1}{2}$.

Generalizations to the case that instead of the scalar diffusion constant $e^{u}$ in (1.1), we have a diffusion matrix $A=\left(a_{i j}\right)_{i, j=1, \ldots, d}$ with

$$
\lambda(A)|\xi|^{2} \leq \sum_{i, j=1}^{d} a_{i j} \xi_{i} \xi_{j} \leq \Lambda(A)|\xi|^{2}, \quad \xi \in \mathbb{R}^{d},
$$

for $\lambda(A)>0$ and $\Lambda(A)<\infty$, are possible. In fact, one can prove estimates similar to those of Corollaries 3.2 and 3.5 for $\mathcal{G}:\left(C^{t}(D)\right)^{d \times d} \rightarrow \mathbb{R}^{K}$, and then choose an appropriate prior with $\mu_{0}\left(\left(C^{t}(D)\right)^{d \times d}\right)=1$ in a similar way to the scalar case.

4.2. Weak approximation in a Fourier basis. Let $x=\left(x_{1}, \ldots, x_{d}\right) \in \mathbb{T}^{d}$. With $\varphi_{k_{1}, \ldots, k_{d}}=\mathrm{e}^{i\left(k_{1} x_{1}+\cdots+k_{d} x_{d}\right)}$, the set $\left\{\varphi_{k_{1}, \ldots, k_{d}}\right\}_{k_{1}, \ldots, k_{d} \in \mathbb{Z}}$ forms a basis for $L^{2}\left(\mathbb{T}^{d}\right)$. Define

$$
W^{N}=\operatorname{span}\left\{\varphi_{k_{1}, \ldots, k_{d}},\left|k_{1}\right| \leq N, \ldots,\left|k_{d}\right| \leq N\right\}
$$

and recall the notation established in subsection 2.3. ${ }^{1}$ We let $p$ denote the $H^{1}$ valued random variable found from the solution of the elliptic problem (1.1) with $u$ distributed according to the posterior $\mu^{y}$ and $p^{N}$ the analogous random variable with $u=u_{F}^{N}$ distributed according to $\nu^{N}$ with the Fourier truncation described above. We have the following approximation theorem.

THEOREM 4.2. Consider the inverse problem of finding u from noisy observations of $p$ in the form of (1.3) and with $p$ solving (1.1) and $D=(-\pi, \pi)^{d}$. Let Assumption 3.6 hold and consider $\mu_{0}$ to be distributed as $\mathcal{N}\left(0,(-\Delta)^{-s}\right)$ with $\Delta$ the Laplacian operator acting on $H^{2}\left(\mathbb{T}^{d}\right)$ Sobolev functions with zero average on $\mathbb{T}^{d}$, and $s>d / 2$. Then, for any $t<s-\frac{d}{2}$,

$$
\left\|\mathbb{E}^{\mu^{y}} p-\mathbb{E}^{\nu^{N}} p^{N}\right\|_{H^{1}} \leq C N^{-t}
$$

\footnotetext{
${ }^{1}$ The slightly different interpretation of $N$ should not cause any confusion in what follows.
} 
and, with $\bar{p}=\mathbb{E}^{\mu^{y}} p, \bar{p}^{N}=\mathbb{E}^{\nu^{N}} p^{N}$, and $S=H_{0}^{1} \otimes H_{0}^{1}$, we have

$$
\left\|\mathbb{E}^{\mu^{y}}(p-\bar{p}) \otimes(p-\bar{p})-\mathbb{E}^{\nu^{N}}\left(p^{N}-\bar{p}^{N}\right) \otimes\left(p^{N}-\bar{p}^{N}\right)\right\|_{S} \leq C N^{-t} .
$$

Note that the mean and covariance of the pressure field, when conditioned by the data, may be viewed as giving a quantification of uncertainty in the pressure when conditioned on data. The previous theorem thus estimates errors arising in this quantification of uncertainty when Karhunen-Loève truncation is used to represent the posterior measure.

Proof of Theorem 4.2. We apply Theorem 2.6 with $G(u)=p$ and $S=H_{0}^{1}(D)$ or $G(u)=p \otimes p$ and $S=H_{0}^{1} \otimes H_{0}^{1}$. We choose $X^{\prime}=X^{\prime \prime}=L^{\infty}$ and $X=C^{t}$ for any $t<s-\frac{d}{2}$. Then $X$ is continuously embedded into $X^{\prime}$ and $X^{\prime}$ into $X^{\prime \prime}$ and, under the assumptions of the theorem, $\mu_{0}\left(X^{\prime \prime}\right)=\mu_{0}\left(X^{\prime}\right)=\mu_{0}(X)=1$. From Proposition 3.1 it is straightforward to show the required Lipschitz condition on $G$ (in either the pressure or pressure covariance cases) whilst the required Lipschitz condition on $\mathcal{G}$ follows from Assumptions 3.6. It remains to prove the operator norm bounds on $P^{\perp}$. We consider dimension $d=2$ first. We write

$$
\begin{aligned}
u_{F}^{N}(x, y) & =\sum_{-N \leq k \leq N} \sum_{-N \leq j \leq N} \hat{u}(j, k) \mathrm{e}^{i(x j+k y)} \\
& =\sum_{-N \leq k \leq N} \sum_{-N \leq j \leq N} \frac{1}{4 \pi^{2}} \int_{-\pi}^{\pi} \int_{-\pi}^{\pi} u(\zeta, \xi) \mathrm{e}^{-i(\zeta j+\xi k)} \mathrm{d} \zeta \mathrm{d} \xi \mathrm{e}^{i(x j+k y)} \\
& =\frac{1}{\pi^{2}} \int_{-\pi}^{\pi} \int_{-\pi}^{\pi} u(\zeta, \xi) D_{N}(x-\zeta) D_{N}(y-\xi) \mathrm{d} \zeta \mathrm{d} \xi \\
& =\frac{1}{\pi^{2}} \int_{-\pi}^{\pi} \int_{-\pi}^{\pi} u(x-\zeta, y-\xi) D_{N}(\zeta) D_{N}(\xi) \mathrm{d} \zeta \mathrm{d} \xi,
\end{aligned}
$$

where, as shown in [28],

$$
D_{N}(x)=\frac{1}{2} \sum_{-N \leq n \leq N} \mathrm{e}^{i x n}=\frac{1}{2} \frac{\sin (N+1 / 2) x}{\sin (x / 2)} .
$$

Noting that $\int_{-\pi}^{\pi} D_{N}(x) \mathrm{d} x=\pi$, we have

$$
\begin{aligned}
u_{F}^{N}(x, y)- & u(x, y)=\frac{1}{\pi^{2}} \int_{-\pi}^{\pi} \int_{-\pi}^{\pi}(u(x-\zeta, y-\xi)-u(x, y)) D_{N}(\zeta) D_{N}(\xi) \mathrm{d} \zeta \mathrm{d} \xi \\
= & \frac{1}{\pi^{2}} \int_{-\pi}^{\pi} \int_{-\pi}^{\pi}(u(x-\zeta, y-\xi)-u(x-\zeta, y) \\
& \quad+u(x-\zeta, y)-u(x, y)) D_{N}(\zeta) D_{N}(\xi) \mathrm{d} \zeta \mathrm{d} \xi \\
= & \frac{1}{\pi^{2}} \int_{-\pi}^{\pi}\left(\int_{-\pi}^{\pi}(u(x-\zeta, y-\xi)-u(x-\zeta, y)) D_{N}(\xi) \mathrm{d} \xi\right) D_{N}(\zeta) \mathrm{d} \zeta \\
& +\frac{1}{\pi^{2}} \int_{-\pi}^{\pi}(u(x-\zeta, y)-u(x, y)) D_{N}(\zeta) \mathrm{d} \zeta \int_{-\pi}^{\pi} D_{N}(\xi) \mathrm{d} \xi
\end{aligned}
$$

To estimate the right-hand side we set $f(\xi)=u(x-\zeta, y-\xi)-u(x-\zeta, y)$ for fixed 
$x, \zeta, y \in \mathbb{T}$, and noting that $f(\xi)$ is periodic we write

$$
\begin{aligned}
2 \int_{-\pi}^{\pi} f(\xi) D_{N}(\xi) \mathrm{d} \xi & =\int_{-\pi}^{\pi} \frac{f(\xi)}{\sin (\xi / 2)} \sin ((N+1 / 2) \xi) \mathrm{d} \xi \\
& =-\int_{-\pi-\frac{\pi}{N+1 / 2}}^{\pi-\frac{\pi}{N+1 / 2}} \frac{f\left(\xi+\frac{\pi}{N+1 / 2}\right)}{\sin \left(\frac{\xi}{2}+\frac{\pi}{2 N+1}\right)} \sin ((N+1 / 2) \xi) \mathrm{d} \xi \\
& =-\int_{-\pi}^{\pi} \frac{f\left(\xi+\frac{\pi}{N+1 / 2}\right)}{\sin \left(\frac{\xi}{2}+\frac{\pi}{2 N+1}\right)} \sin ((N+1 / 2) \xi) \mathrm{d} \xi \\
& =\frac{1}{2} \int_{-\pi}^{\pi}\left(\frac{f(\xi)}{\sin (\xi / 2)}-\frac{f\left(\xi+\frac{\pi}{N+1 / 2}\right)}{\sin \left(\frac{\xi}{2}+\frac{\pi}{2 N+1}\right)}\right) \sin ((N+1 / 2) \xi) \mathrm{d} \xi
\end{aligned}
$$

In the third line we have used $\int_{-\pi-\alpha}^{-\pi} g(\xi) \mathrm{d} \xi=\int_{\pi-\alpha}^{\pi} g(\xi) \mathrm{d} \xi$ for any $2 \pi$ periodic function $g$ and any $\alpha \in \mathbb{R}$. Let $h=\frac{2 \pi}{2 N+1}$. We can write

$$
\begin{aligned}
4 \int_{-\pi}^{\pi} f(\xi) D_{N}(\xi) \mathrm{d} \xi & \leq \int_{-\pi}^{\pi}\left|\frac{f(\xi)}{\sin (\xi / 2)}-\frac{f(\xi+h)}{\sin \left(\frac{\xi+h}{2}\right)}\right| \mathrm{d} \xi \\
& =I_{1}+I_{2}+I_{3}
\end{aligned}
$$

with $I_{1}=\int_{0}^{\pi}, I_{2}=\int_{-\pi}^{-h}$, and $I_{3}=\int_{-h}^{0}$ of the integrand in the right-hand side of the above inequality. Noting that $f(0)=0$, we have

$$
\begin{aligned}
I_{1} & =\int_{0}^{\pi}\left|\frac{f(\xi)-f(\xi+h)}{\sin \left(\frac{\xi+h}{2}\right)}+f(\xi)\left(\frac{1}{\sin (\xi / 2)}-\frac{1}{\sin \left(\frac{\xi+h}{2}\right)}\right)\right| \mathrm{d} \xi \\
& \leq c h^{t}\|f\|_{C^{t}} \int_{0}^{\pi} \frac{1}{\xi+h} \mathrm{~d} \xi+\int_{0}^{\pi} \frac{\|f\|_{C^{t}} \xi^{t}(\sin (\xi / 2+h / 2)-\sin (\xi / 2))}{\sin (\xi / 2) \sin (\xi / 2+h / 2)} \mathrm{d} \xi \\
& \leq c h^{t}\|f\|_{C^{t}} \log \frac{1}{h}+c h \int_{0}^{\pi} \frac{\|f\|_{C^{t}} \xi^{t}}{\xi(\xi+h)} \mathrm{d} \xi \\
& \leq c h^{t}\|f\|_{C^{t}} \log \frac{1}{h}+c h\|f\|_{C^{t}} \int_{0}^{h} \frac{1}{\xi^{1-t} h}+c h\|f\|_{C^{t}} \int_{h}^{\pi} \frac{1}{h^{1-t}(\xi+h)} \\
& \leq c h^{t}\|f\|_{C^{t}} \log \frac{1}{h}+c h^{t}\|f\|_{C^{t}} .
\end{aligned}
$$

Similarly

$$
\begin{aligned}
I_{2} & =\int_{-\pi}^{-h}\left|\frac{f(\xi)-f(\xi+h)}{\sin \left(\frac{\xi}{2}\right)}-f(\xi+h)\left(\frac{1}{\sin \left(\frac{\xi+h}{2}\right)}-\frac{1}{\sin (\xi / 2)}\right)\right| \mathrm{d} \xi \\
& \leq c h^{t}\|f\|_{C^{t}} \int_{-\pi}^{-h} \frac{1}{-\xi} \mathrm{d} \xi+\int_{-\pi}^{-h} \frac{\|f\|_{C^{t}}|\xi+h|^{t}|\sin (\xi / 2)-\sin ((\xi+h) / 2)|}{|\sin (\xi / 2)||\sin ((\xi+h) / 2)|} \mathrm{d} \xi \\
& \leq c h^{t}\|f\|_{C^{t}} \log \frac{1}{h}+c h \int_{-\pi}^{-h} \frac{\|f\|_{C^{t}}|\xi+h|^{t}}{|\xi||\xi+h|} \mathrm{d} \xi \\
& \leq c h^{t}\|f\|_{C^{t}} \log \frac{1}{h}+c h\|f\|_{C^{t}} \int_{-\pi}^{-2 h} \frac{1}{|\xi| h^{1-t}} \mathrm{~d} \xi+c h\|f\|_{C^{t}} \int_{-2 h}^{-h} \frac{1}{h|\xi+h|^{1-t}} \mathrm{~d} \xi \\
& \leq c h^{t}\|f\|_{C^{t}} \log \frac{1}{h}+c h^{t}\|f\|_{C^{t}} .
\end{aligned}
$$

Copyright (c) by SIAM. Unauthorized reproduction of this article is prohibited. 
Finally

$$
\begin{aligned}
I_{3} & \leq c \int_{-h}^{0}\left|\frac{f(\xi)}{\xi}\right|+\left|\frac{f(\xi+h)}{\xi+h}\right| \mathrm{d} \xi \\
& \leq c\|f\|_{C^{t}} \int_{-h}^{0} \frac{1}{|\xi|^{1-t}} \mathrm{~d} \xi+c\|f\|_{C^{t}} \int_{-h}^{0} \frac{1}{|\xi+h|^{1-t}} \mathrm{~d} \xi \\
& \leq c h^{t}\|f\|_{C^{t}} .
\end{aligned}
$$

Hence we have

$$
\int_{-\pi}^{\pi}(u(x-\zeta, y-\xi)-u(x-\zeta, y)) D_{N}(\xi) \mathrm{d} \xi \leq C N^{-t}\|u\|_{C^{t}} \log N
$$

and

$$
\int_{-\pi}^{\pi}(u(x-\zeta, y)-u(x, y)) D_{N}(\zeta) \mathrm{d} \zeta \leq C N^{-t}\|u\|_{C^{t}} \log N
$$

Now since for fixed $\epsilon>0$ sufficiently small,

$$
\begin{aligned}
\int_{0}^{\pi} \frac{|\sin (N+1 / 2) x|}{|\sin (x / 2)|} \mathrm{d} x & \leq \int_{0}^{\epsilon / N}(2 N+1) \mathrm{d} x+\int_{\epsilon / N}^{\epsilon} \frac{1}{x / 2} \mathrm{~d} x+\int_{\epsilon}^{\pi} \frac{|\sin (N+1 / 2) x|}{|\sin (x / 2)|} \mathrm{d} x \\
& \leq\left(2+\frac{1}{N}\right) \epsilon+2 \log N+C(\epsilon) \\
& \leq c \log N \quad \text { as } N \rightarrow \infty,
\end{aligned}
$$

we have $\left\|D_{N}(\xi)\right\|_{L^{1}(-\pi, \pi)}=O(\log N)$ and therefore

$$
\left|u_{F}^{N}(x, y)-u(x, y)\right| \leq C\|u\|_{C^{t}(D)} N^{-t}(\log N)^{2} \quad \text { for any } x, y \in \mathbb{T}^{2} .
$$

Similarly in the three-dimensional case one can show that

$$
\left\|u_{F}^{N}-u\right\|_{L^{\infty}(D)} \leq C\|u\|_{C^{t}(D)} N^{-t}(\log N)^{3} .
$$

Since $t$ can be chosen arbitrarily close to $s-\frac{d}{2}$ we obtain $\left\|P^{\perp}\right\|_{\mathcal{L}\left(X, X^{\prime \prime}\right)}=\mathcal{O}\left(N^{-t}\right)$ for any $t<s-\frac{d}{2}$. This, since $X^{\prime}=X^{\prime \prime}$, implies that $\left\|P^{\perp}\right\|_{\mathcal{L}\left(X, X^{\prime}\right)}$ is bounded independently of $N$. The result follows by Theorem 2.6.

5. Conclusion. We have addressed the inverse problem of finding the diffusion coefficient in a uniformly elliptic PDE in divergence form, when noisy observations of its solution are given, using a Bayesian approach. We have applied the results of $[8,27]$ on well definedness and well posedness of the posterior measure to show that for an appropriate choice of prior measure this inverse problem is well posed. We also provided a general theorem concerning weak approximation of the posterior using finite-dimensional truncation of the Karhunen-Loève expansion: Theorem 2.6. We have then used the result of this theorem to give an estimate of the weak error in the posterior measure when using Fourier truncation: Theorem 4.2. Future work arising from the results in this paper includes the possibility of application to other inverse problems, the study of rare events and the effect of approximation, and the question of obtaining improved rates of weak convergence under stronger conditions on the mapping $G$. Also of interest is the extension to non-Gaussian priors of Besov type [18], which is considered in [13]. 
Appendix A. Let $D \subset \mathbb{R}^{d}$ be open and bounded and let $p \in W_{0}^{1, q}(D)$ satisfy the following integral identity:

$$
\int_{D} \nabla v \cdot(a \nabla p+e)+f v \mathrm{~d} x=0
$$

for any $v \in C_{0}^{1}(D)$ and with $a$ satisfying $0<\lambda \leq a \leq \Lambda<\infty$. We find an estimate for the $W^{1, q}$ norm of $p$ with special attention on how the upper bound depends on the diffusion coefficient $a$. The results of this appendix are obtained using slight modification of the proof of Shaposhnikov [26] for our purpose here.

In the following, Lemma A.1 gives an estimate for the $W^{1, q}$ norm of $p$ over $B$, a ball of sufficiently small radius in $\mathbb{R}^{d}$ and of center $0 \in \mathbb{R}^{d}$. Lemma A.2 gives a similar result for the case that the domain is $B \cap\left\{\left(x^{1}, \ldots, x^{d}\right) \in \mathbb{R}^{d}: x^{d} \geq 0\right\}$. This lemma allows us to consider the effect of the boundary when generalizing to a bounded domain D. Lemma A.1 and A.2 are then used to prove Theorem A.3 which gives an estimate for $\|p\|_{W^{1, q}(D)}$ in a general bounded domain $D$. Finally in Corollary A.4 we consider $a \in C^{t}(D)$ and obtain an estimate for $\|p\|_{W^{1, q}}$ with a polynomial dependence on $\|a\|_{C^{t}(D)}$.

Notation. In this appendix we use the operator $P_{r}$, which for $g$ a scalar function is defined as

$$
P_{r}(g)(y)=\int_{B(0, r)} K(x-y) g(x) \mathrm{d} x
$$

where

$$
K(y)= \begin{cases}\frac{-1}{d(d-1) \alpha(d)|y|^{d-2}}, & d>2 \\ \frac{1}{2 \pi} \ln |y|, & d=2 .\end{cases}
$$

Also for any $r>0$ we define

$$
B(0, r)=\left\{x \in \mathbb{R}^{d}:|x|<r\right\} .
$$

Lemma A.1. Let $a \in C(B(0,1))$ and $a(0)=a_{0}$. Suppose that for a positive $r<1, p \in W_{0}^{1, q}(B(0, r))$ satisfies (A.1) with $D \equiv B(0, r), P_{r} f \in W^{1, q}(B(0, r))$, $e \in L^{q}(B(0, r))$, and supp $p$, supp $f$, supp $e \subset B(0, r)$. Then, letting $B=B(0, r)$, we have

$$
\|p\|_{W^{1, q}(B)} \leq \frac{1}{\lambda} \frac{C_{0}}{C_{1}}(1+r)\left(\|e\|_{L^{q}(B)}+\left\|P_{r}(f)\right\|_{W^{1, q}(B)}\right),
$$

with $C_{0}$ a constant depending only on $d$ and $q$ and

$$
C_{1}=1-\frac{(1+r) C_{0}}{\lambda} \sup _{B}\left|a-a_{0}\right|>0
$$

Lemma A.2. Let $a \in C(B(0,1)), a(0)=a_{0}$, and $G(0, r)=B(0, r) \cap\left\{\left(x^{1}, \ldots, x^{d}\right)\right.$ : $\left.x^{d} \geq 0\right\}$ for positive $r<1$. Suppose that $p \in W_{0}^{1, q}(G(0, r))$ satisfies (A.1) with $D \equiv G(0, r), P_{r} f \in W^{1, q}(G(0, r))$, and $e \in L^{q}(G(0, r))$. Assume that the functions $p, f$, and e vanish in a neighborhood of the spherical part of the hemisphere $G(0, r)$ but not necessarily on $G(0, r) \cap\left\{x^{d}=0\right\}$. Then the result of Lemma A.1 with $B=G(0, r)$ holds.

Note that although in both Lemmas A.1 and A.2, $p$ vanishes on the boundary of $B(0, r)$ and $G(0, r)$, respectively, in Lemma A.2 the support of $p$ need not be the subset of an open subset of $G(0, r)$. The above results follow from the proofs of Lemmas 2 and 3 of [26]. 
Theorem A.3. Assume that $D \subset \mathbb{R}^{d}, d=2,3$, is a bounded $C^{1}$ domain, and $a \in C(\bar{D})$. Suppose also that $e \in L^{q}(D)$ and $f \in L^{2}(D)$. Then for $2<q<6$

$$
\|p\|_{W^{1, q}} \leq \frac{C(D, d, q)}{\lambda}\left(1+\frac{1}{\delta^{1+d}}\right)(1+\delta)(1+\Lambda)\left(\|e\|_{L^{q}(D)}+\|f\|_{L^{2}(D)}\right),
$$

where $\delta$ is a positive constant that satisfies the following:

$$
\max _{y \in B(x, \delta)}|a(y)-a(x)| \leq \frac{\lambda}{4 C_{0}(d, q)} \quad \text { for any } x \in D,
$$

where $C_{0}(d, q)$ is the constant in Lemma A.1.

Proof. Choose $r_{D}$ so that for any $x \in D$ there exists a ball of radius $r_{D}$ inside $D$ that contains $x$. Let $r=\min \left\{r_{D}, \delta\right\}$. Corresponding to $r$, consider $\left\{x_{j}\right\}_{j=1}^{J} \subset \bar{D}$ so that the set of the neighborhoods of these points, $\left\{U\left(x_{j}\right)\right\}_{j=1}^{J}$ defined as follows, forms a cover of $\bar{D}$ :

- for $x_{j} \in D, U\left(x_{j}\right)=B\left(x_{j}, r\right)$,

- for $x_{j} \in \partial D, U\left(x_{j}\right)=B\left(x_{j}, r\right) \cap \bar{D}$ and there exists $C^{1}$ mapping $\psi_{j}$ such that $\psi_{j}\left(U\left(x_{j}\right)\right)=G\left(x_{j}, r\right)$, where $G\left(x_{j}, r\right)=B\left(x_{j}, r\right) \cap\left\{\left(x^{1}, \ldots, x^{d}\right): x^{d} \geq x_{j}^{d}\right\}$,

- and there exists a partition of unity $\left\{\xi_{j}\right\}_{j=1}^{J}$ subordinate to $\left\{U\left(x_{j}\right)\right\}$.

Let $w_{j}=\xi_{j} p$. Hence $p=\sum_{j=1}^{J} w_{j}$. In the following we will apply the result of Lemmas A.1 and A.2 to estimate $\left\|\xi_{j} p\right\|_{W^{1, q}}$, which then results in an estimate for $\|p\|_{W^{1, q}} \leq \sum_{j=1}^{J}\left\|w_{j}\right\|_{W^{1, q}}$. Define

$$
\tilde{e}=\xi_{j} e-a p \nabla \xi_{j} \quad \text { and } \quad \tilde{f}=\xi_{j} f+a \nabla \xi_{j} \cdot \nabla p .
$$

On $U\left(x_{j}\right)$ with $x_{j} \in D, w_{j}$ satisfies

$$
\int_{B\left(x_{j}, r\right)} \nabla v \cdot\left(a \nabla w_{j}+\tilde{e}\right)+v \tilde{f} \mathrm{~d} x=0 .
$$

By Lemma A.1, with $B_{j}=B\left(x_{j}, r\right)$, we have

$$
\left\|w_{j}\right\|_{W^{1, q}\left(B_{j}\right)} \leq \frac{C(d, q)}{\lambda}(1+r)\left(\|\tilde{e}\|_{L^{q}\left(B_{j}\right)}+\left\|P_{r}(\tilde{f})\right\|_{W^{1, q}\left(B_{j}\right)}\right) .
$$

To estimate $\|\tilde{e}\|_{L^{q}\left(B_{j}\right)}$ we write

$$
\begin{aligned}
\|\tilde{e}\|_{L^{q}\left(B_{j}\right)} & \leq\left\|\xi_{j} e\right\|_{L^{q}\left(B_{j}\right)}+\left\|a \nabla \xi_{j} p\right\|_{L^{q}\left(B_{j}\right)} \\
& \leq(1+\Lambda) C\left(\xi_{j}, \nabla \xi_{j}\right)\left(\|e\|_{L^{q}}+\|p\|_{\left.L^{q}\right)}\right. \\
& \leq \frac{c(1+\Lambda)}{r}\left(\|e\|_{L^{q}}+\|p\|_{L^{q}}\right) .
\end{aligned}
$$

For $\left\|P_{r}(\tilde{f})\right\|_{W^{1, q}\left(B_{j}\right)}$, by Sobolev embedding theorem (assuming that $2 \leq q \leq 6$ ) and since by Theorem 9.9 of $[17]\left\|P_{r} g\right\|_{H^{2}} \leq C(d)\|g\|_{L^{2}}$, we have

$$
\begin{aligned}
\left\|P_{r}(\tilde{f})\right\|_{W^{1, q}(D)} & \leq C(D)\left\|P_{r}(\tilde{f})\right\|_{H^{2}} \\
& \leq C(D, d)\|\tilde{f}\|_{L^{2}} \\
& \leq C(D, d)\left(\left\|\xi_{j} f\right\|_{L^{2}}+\left\|a \nabla \xi_{j} \cdot \nabla p\right\|_{L^{2}}\right) \\
& \leq C\left(D, d, \xi_{j}, \nabla \xi_{j}\right)(1+\Lambda)\left(\|f\|_{L^{2}}+\|\nabla p\|_{L^{2}}\right) \\
& \leq \frac{C(D, d)(1+\Lambda)}{r}\left(\|f\|_{L^{2}}+\|\nabla p\|_{L^{2}}\right) .
\end{aligned}
$$

Since $2<q<6$ and $D$ is bounded,

$$
\|p\|_{L^{q}} \leq c\|\nabla p\|_{L^{2}} \leq c\left(\|f\|_{L^{2}}+\|e\|_{L^{2}}\right)
$$

Copyright $@$ by SIAM. Unauthorized reproduction of this article is prohibited. 
where the second inequality is obtained by taking the inner product of (A.1) with $p$ (noting that $p \in W_{0}^{1, q}(D)$ and hence $\left.p\right|_{\partial D}=0$ in the trace sense). Therefore

$$
\left\|w_{j}\right\|_{W^{1, q}\left(B_{j}\right)} \leq \frac{C(D, d, q)}{r \lambda}(1+\Lambda)(1+r)\left(\|e\|_{L^{q}}+\|f\|_{L^{2}}\right) .
$$

It remains to consider the case that $x_{j} \in \partial D$. For such $x_{j}, w_{j}$ on $U\left(x_{j}\right)$ satisfies (using the map $\psi_{j}$ defined at the beginning of the proof)

$$
\int_{G\left(x_{j}, r\right)} \nabla v \cdot\left(\hat{a} \nabla w_{j}+\hat{e}\right)+v \hat{f} \mathrm{~d} x=0
$$

where $\hat{a}, \hat{e}$, and $\hat{f}$ depend on $\nabla \psi_{j}$. It is not difficult to see that $\left|\nabla \psi_{j}\right|<C$ where $C$ depends only on the properties of the boundary of $D$, therefore in a similar way to the above argument and using Lemma A.2 it can be shown that

$$
\left\|w_{j}\right\|_{W^{1, q}\left(G_{j}\right)} \leq \frac{C(D, d, q)}{r \lambda}(1+\Lambda)(1+r)\left(\|e\|_{L^{q}}+\|f\|_{L^{2}}\right) .
$$

Now we can write

$$
\begin{aligned}
\|p\|_{W^{1, q}} & \leq \sum_{j=1}^{J}\left\|w_{j}\right\|_{W^{1, q}} \leq \frac{c|D|}{r^{d}}\left\|w_{j}\right\|_{W^{1, q}} \\
& \leq \frac{C(D, d, q)}{r^{1+d} \lambda}(1+\Lambda)(1+r)\left(\|e\|_{L^{q}}+\|f\|_{L^{2}}\right) \\
& \leq \frac{C(D, d, q)}{\lambda}\left(1+\frac{1}{\delta^{1+d}}\right)\left(1+\frac{1}{r_{D}^{1+d}}\right)(1+\Lambda)\left(1+r_{D}\right)(1+\delta)\left(\|e\|_{L^{q}}+\|f\|_{L^{2}}\right) \\
& \leq \frac{C(D, d, q)}{\lambda}\left(1+\frac{1}{\delta^{1+d}}\right)(1+\Lambda)(1+\delta)\left(\|e\|_{L^{q}}+\|f\|_{L^{2}}\right)
\end{aligned}
$$

and the result follows.

In order to quantify $\delta$ in the above theorem, in terms of the norm of the space that $a$ lives in, we need to assume $a$ to be Hölder continuous.

Corollary A.4. Suppose that the assumptions of Theorem A.3 hold. Assume also that a is $t$-Hölder continuous in $D$. Then

$$
\leq \frac{C(D, d, q, t)}{\lambda}\left(1+\frac{\|a\|_{C^{t}(D)}^{(1+d) / t}}{\lambda^{(1+d) / t}}\right)\left(1+\frac{\|a\|_{C^{t}(D)}^{1 / t}}{\lambda^{1 / t}}\right)(1+\Lambda)\left(\|e\|_{L^{q}(D)}+\|f\|_{L^{2}(D)}\right) .
$$

Proof. Since $|a(x)-a(y)| \leq\|a\|_{C^{t}}|x-y|^{t}, \delta$ of Theorem A.3 satisfies $\delta \leq$ $c \lambda^{1 / t}\|a\|_{C^{t}(D)}^{-1 / t}$ and the result follows.

Acknowledgments. The authors are grateful to Jose Rodrigo and Christoph Schwab for helpful discussions.

\section{REFERENCES}

[1] R. A. Adams, Sobolev Spaces, Pure Appl. Math. 65, Academic Press, New York, London, 1975.

[2] I. Babuška, F. Nobile, And R. Tempone, A stochastic collocation method for elliptic partial differential equations with random input data, SIAM J. Numer. Anal., 45 (2007), pp. 10051034 .

Copyright $@$ by SIAM. Unauthorized reproduction of this article is prohibited. 
[3] I. Babuška, R. Tempone, And G. E. Zouraris, Galerkin finite element approximations of stochastic elliptic partial differential equations, SIAM J. Numer. Anal., 42 (2004), pp. 800825.

[4] M. Bieri And C. Schwab, Sparse high order FEM for elliptic sPDEs, Comput. Methods Appl. Mech. Engrg., 198 (2009), pp. 1149-1170.

[5] V. I. Bogachev, Gaussian Measures, Math. Surveys Monogr. 62, American Mathematical Society, Providence, RI, 1998.

[6] J. CHARRIER, Strong and weak error estimates for elliptic partial differential equations with random coefficients, submitted.

[7] A. Cohen, R. DeVore, and C. Schwab, Analytic regularity and polynomial approximation of parametric and stochastic elliptic PDEs, Anal. Appl. (Singap.), 9 (2011), pp. 11-47.

[8] S. L. Cotter, M. Dashti, J. C. Robinson, and A. M. Stuart, Bayesian inverse problems for functions with applications in fluid mechanics, Inverse Problems, 25 (2009), 115008.

[9] S. L. Cotter, M. Dashti, And A. M. Stuart, Approximation of Bayesian inverse problems in differential equations, SIAM J. Numer. Anal., 48 (2010), pp. 322-345.

[10] S. L. Cotter, M. Dashti, And A. M. Stuart, Variational data assimilation using targeted random walks, Int. J. Numer. Methods Fluids, to appear.

[11] S. L. Cotter, G. O. Roberts, A. M. Stuart, and D. White, MCMC methods for functions: Modifying old algorithms to make them faster, submitted.

[12] G. Da Prato And J. ZabczyK, Stochastic Equations in Infinite Dimensions, Encyclopedia Math. Appl. 44, Cambridge University Press, Cambridge, UK, 1992.

[13] M. Dashti, S. Harris, AND A. M. Stuart, Besov priors for Bayesian inverse problems, submitted.

[14] M. Dashti, K. J. H. Law, A. M. Stuart, and J. Voss, MAP estimators for Bayesian nonparametrics, in preparation.

[15] R. M. Dudley, Real Analysis and Probability, 2nd ed., Cambridge University Press, UK, 2002.

[16] P. Frauenfelder, C. Schwab, And R. A. Todor, Finite elements for elliptic problems with stochastic coefficients, Comput. Methods Appl. Mech. Engrg., 194 (2005), pp. 205-228.

[17] D. Gilbarg and N. S. Trudinger, Elliptic Partial Differential Equations of Second Order, Springer-Verlag, Berlin, 1983.

[18] M. Lassas, E. Saksman, and S. Siltanen, Discretization-invariant Bayesian inversion and Besov space priors, Inverse Prob. Imaging, 3 (2009), pp. 87-122.

[19] M. A. Lifshits, Gaussian Random Functions, Math. Appl. 322, Kluwer Academic Publishers, Dordrecht, The Netherlands, 1995.

[20] H. G. Matthies, Stochastic finite elements: Computational approaches to stochastic partial differential equations, ZAMM Z. Angew. Math. Mech., 88 (2008), pp. 849-873.

[21] H. G. Matthies And C. Bucher, Finite elements for stochastic media problems, Comput. Methods Appl. Mech. Engrg., 168 (1999), pp. 3-17.

[22] D. McLaughlin and L. Townley, A reassessment of the ground water inverse problem, Water Resour. Res., 32 (1996), pp. 1131-1161.

[23] F. Nobile, R. Tempone, and C. G. Webster, An anisotropic sparse grid stochastic collocation method for partial differential equations with random input data, SIAM J. Numer. Anal., 46 (2008), pp. 2411-2442.

[24] F. Nobile, R. Tempone, And C. G. Webster, A sparse grid stochastic collocation method for partial differential equations with random input data, SIAM J. Numer. Anal., 46 (2008), pp. 2309-2345.

[25] C. Schwab And R. A. Todor, Karhunen-Loève approximation of random fields by generalized fast multipole methods, J. Comput. Phys., 217 (2006), pp. 100-122.

[26] S. V. Shaposhnikov, On Morrey's estimate for the Sobolev norms of solutions of elliptic equations, (Russian) Mat. Zametki, 79 (2006), pp. 450-469; translation in Math. Notes, 79 (2006), pp. 413-430.

[27] A. M. StuART, Inverse problems: A Bayesian perspective, Acta Numer., 19 (2010), pp. 451-559.

[28] A. Zygmund, Trigonometric Series. Vol. I, II. Reprint of the 1979 edition. Cambridge Math. Lib., Cambridge University Press, Cambridge, UK, 1988.

[29] D. Zhang AND Z. LU, An efficient, high-order perturbation approach for flow in random porous media via Karhunen-Loève and polynomial expansions, J. Comput. Phys., 194 (2004), pp. $773-794$.

Copyright (c) by SIAM. Unauthorized reproduction of this article is prohibited. 